\title{
Macrolide susceptibility in Streptococcus pyogenes: monitoring of isolates collected from throat swabs for the period november 2003 to september 2006
}

\author{
Maria Gabriella Mazzarello', Rinaldo Brunetti', Mascja Perfumo', Simona Roveta², \\ Anna Marchese ${ }^{2}$ \\ 'Laboratorio Analisi Chimico Cliniche e Microbiologiche ASL 22, P.O. di Ovada, via Ruffini 22, I 5076 Ovada (AL). \\ ${ }_{2}^{2}$ Sezione di Microbiologia, DISCAT - Università di Genova, Largo R. Benzi 10, I6I32 Genova.
}

Key words: Streptococcus pyogenes, macrolides, resistance.

Sensibilità ai macrolidi di Streptococcus pyogenes: monitoraggio degli isolati provenienti da tampone faringeo nel periodo novembre 2003-settembre 2006

\section{SUMMARY}

Background: Streptococcus pyogenes can cause acute suppurative infections (tonsillitis and pharyngitis) and serious complications like rheumatic fever or acute post-streptococcal glomerulonephritis. Antibiotic treatment of streptococcal infections is recommended and penicillin is the therapy of choice, moreover, macrolides represent an important alternative particularly for patients with $\beta$-lactam-associated allergies. However, increasing resistance of S. pyogenes to macrolides has been observed in some European countries (especially in Spain and Italy) in the last decade. This study was conducted to update our knowledge on this evolution.

Materials and methods: A total of 128 clinical strains of S. pyogenes isolated from 933 throat swabs collected in the Clinical Microbiological Laboratory of Ovada (ASL 22) between November 2003 and September 2006 were tested for their susceptibility to erythromycin, clindamycin and rokitamycin. The phenotype of macrolide resistance was determined by the triple-disc diffusion test method (Giovanetti et al, 1999).

Results: S. pyogenes strains isolated between November 2003 and December 2005 showed a percentage of macrolide-resistance of 15\%; more precisely $9 \%$ with phenotype $M$ and $6 \%$ with phenotype iMLS. Only 3 of the 28 isolates (10.7\%) collected between January-September 2006 were macrolide-resistant (phenotype M).All I28 isolates were fully susceptible to rokitamycin.

Conclusion: Macrolide resistance rates determined in the present study were relatively low in comparison to the values registered in our country in the last decade. Efflux of the drug (phenotype $M$ ) is the predominating mechanism. This type of resistance does not affect the activity of macrolides 16-membered (e.g. rokitamicin). However, changes of resistance rates and their prevailing mechanisms can occur rapidly. For safe empirical prescription of macrolides, further surveillance studies that include the identification of resistance mechanisms are required to follow current trends.

Received February I, 2007

Accepted April 26, 2007

\section{INTRODUZIONE}

Streptococcus pyogenes è il principale agente eziologico batterico responsabile di faringo-tonsilliti acute. Questo patogeno può causare anche gravi complicanze come la febbre reumatica e la glomerulonefrite acuta $(18,23)$. La penicillina resta l'antibiotico di scelta per la terapia delle infezioni streptococciche, mentre i macrolidi rappresentano, specie nei soggetti con ipersensibilità ai beta-lattamici, il trattamento alternativo più diffuso $(9,11,24)$. Sebbene $S$. pyogenes mantenga tuttora una sensibilità in vitro del $100 \%$ nei confronti della penicillina, è possibile che in alcuni casi l'antibiotico non riesca a produrre l'eradicazione del patogeno. È stato infatti dimostrato che alcuni stipiti di Streptococco di gruppo A possono penetrare all'interno delle cellule dell'epitelio respiratorio sfuggendo quindi all'azione di quegli antibiotici, come la penicillina, incapaci di attraversare la membrana cellulare (25). Questa scoperta e il concomitante incremento dell'incidenza di patogeni come Mycoplasma pneumoniae e Chlamydia pneumoniae hanno favorito un utilizzo clinico molto diffuso dei macrolidi (10). Negli ultimi anni, tuttavia, si è assistito in vari paesi europei (inclusa l'Italia) ad un significativo 
aumento della resistenza di $S$. pyogenes nei confronti di questa classe di farmaci $(3,8,15,17,9)$. La resistenza ai macrolidi può manifestarsi attraverso meccanismi differenti, i più comuni sono l'alterazione della subunità ribosomale $50 \mathrm{~S}$ (con conseguente ridotta affinità nei confronti del farmaco) oppure l'efflusso attivo dell'antibiotico dalla cellula $(1,21)$. La modificazione enzimatica del ribosoma batterico avviene ad opera di metilasi codificate da geni erm e la resistenza si può esprimere con fenotipo inducibile (iMLS) o costitutivo (cMLS). La presenza dei geni erm conferisce sempre resistenza ad alto livello a tutti i macrolidi a 14 e 15 atomi e, se la metilasi è prodotta costitutivamente, si può avere anche resistenza alle molecole a 16 atomi, lincosamidi, streptogramine. Il gene mef, largamente diffuso nei ceppi di $S$. pyogenes, conferisce tramite un meccanismo di efflusso resistenza alle molecole a 14 e 15 atomi ma non a macrolidi a 16 atomi, lincosamidi o streptogramine (fenotipo M). I determinanti genetici della resistenza ai macrolidi si trovano in plasmidi e trasposoni spesso associati con geni veicolanti altre resistenze antibiotiche. Alcuni ceppi possono albergare contemporaneamente geni erm e mef, in quanto la presenza dell'uno non condiziona l'acquisizione dell' altro. (26-28, 30, 32, 33).

Questo studio epidemiologico locale si propone di stimare l'incidenza di S. pyogenes sul totale dei tamponi faringei eseguiti nel periodo di tempo preso in esame (considerando sia la popolazione pediatrica sia quella adulta) e monitorare la resistenza manifestata da questo patogeno nei confronti dei macrolidi a 14 e a 16 atomi.

\section{MATERIALI E METODI}

Nel periodo Novembre 2003 - Settembre 2006 presso il Laboratorio Analisi Chimico Cliniche e Microbiologiche dell'ASL 22 (Acqui Terme, Novi Ligure, Ovada), P.O. di Ovada, S.S. di Microbiologia, sono stati eseguiti, sia su pazienti ospedalizzati sia di provenienza comunitaria, 933 tamponi orofaringei per la ricerca di Streptococcus pyogenes.

La semina dei tamponi è stata effettuata su Columbia CNA agar base (bioMérieux Italia S.p.A., Roma, Italy), contenente $10 \mathrm{mg} / 1 \mathrm{di}$ colistina e $15 \mathrm{mg} / \mathrm{l}$ di acido nalidixico che inibiscono la crescita dei germi Gram-negativi, addizionato con $5 \%$ di sangue defibrinato di montone.

$S$. pyogenes forma su questo terreno colonie trasparenti o traslucide con superfici lisce, bordi regolari e con alone di $\beta$-emolisi ben definito. L'appartenenza di ciascun isolato al gruppo A è stata verificata anche tramite il saggio della sensibilità alla bacitracina, impiegando un dischetto da
0,04 U (Mast Group Ltd, Merseyside, UK), posto su una piastra di agar sangue inoculata con il ceppo in esame. Gli isolati su CNA sono stati ulteriormente identificati per il gruppo A mediante test di agglutinazione al lattice (Oxoid S.p.A., Milano, Italy).

I tamponi risultati positivi sono stati classificati in base alla provenienza ed all'età del paziente.

Al fine di determinare il fenotipo di resistenza degli isolati di $\mathrm{S}$. pyogenes verso i macrolidi (cMLS, iMLS oppure M) sono stati impiegati un dischetto di Eritromicina $(15 \mu \mathrm{g})$, uno di Clindamicina $(2 \mu \mathrm{g})$ ed uno di Rokitamicina (30 $\mu \mathrm{g})$. I dischetti sono stati posti a una distanza di $1.5-2 \mathrm{~cm}$ uno dall'altro sulla superficie di una piastra di terreno Mueller-Hinton (MH) agar addizionato con sangue di montone $0.5 \%$ inoculata con una sospensione (torbidità 0.5 McFarland) del ceppo da esaminare $(21,30)$. Dopo 18 ore di incubazione a $37^{\circ} \mathrm{C}$, la comparsa di inibizione attorno al dischetto di clindamicina (diametro $>19 \mathrm{~mm}$ ) indicava il fenotipo $\mathrm{M}$, mentre la deformazione dell'alone di inibizione attorno al dischetto di clindamicina nella zona adiacente a quello di eritromicina indicava il fenotipo iMLS. l'assenza di un alone di inibizione attorno ad entrambi i dischetti indicava invece resistenza costitutiva (cMLS). S. pyogenes ATCC 19615 e S. pneumoniae ATCC 49619 sono stati utilizzati come controlli di qualità.

\section{RISULTATI}

Dei 933 tamponi eseguiti, 128 sono risultati positivi per $S$. pyogenes di gruppo A (con una percentuale pari al $13.7 \%$ ): 65 isolati provenivano da pazienti adulti (2 dei quali ospedalizzati) e $63 \mathrm{da}$ soggetti con età inferiore ai 12 anni (tutti esterni). L'incidenza del patogeno in esame per ciascun anno del periodo di tempo considerato è riportata nella figura I: nel 2004 e 2005 è risultata essere il $16 \%$, con un lieve calo nel $2006(11 \%)$.

Nelle tabelle 1 e 2 sono indicate le percentuali di sensibilità ai macrolidi degli stipiti di S. pyogenes isolati da soggetti adulti e pediatrici (età inferiore ai 12 anni ) nei periodi Novembre 2003-Dicembre 2005 e Gennaio 2006-Settembre 2006. Tutti i 128 ceppi analizzati hanno presentato piena sensibilità alla rokitamicina. I risultati ottenuti nel biennio 2004-2005 hanno mostrato una sensibilità all'eritromicina dell' $85 \%$, superiore nei pazienti adulti $(88.9 \%)$ rispetto a quelli pediatrici $(81.8 \%)$. Tra il $15 \%$ di ceppi che sono risultati resistenti il $9 \%$ presentava fenotipo $\mathrm{M}$ e il $6 \%$ fenotipo iMLS. Il fenotipo $M$ è apparso più diffuso nei pazienti pediatrici ( 8 ceppi su 10 ) rispetto a quelli adulti in cui invece prevaleva il fenotipo iMLS (4 ceppi su 5). Nel 2006 la percentuale di resistenza all'eri- 
tromicina è scesa al $10.7 \%$ con solamente 3 stipiti con fenotipo $\mathrm{M}$ su 28 isolati analizzati, 2 dei quali provenivano da pazienti pediatrici e uno da soggetto adulto.

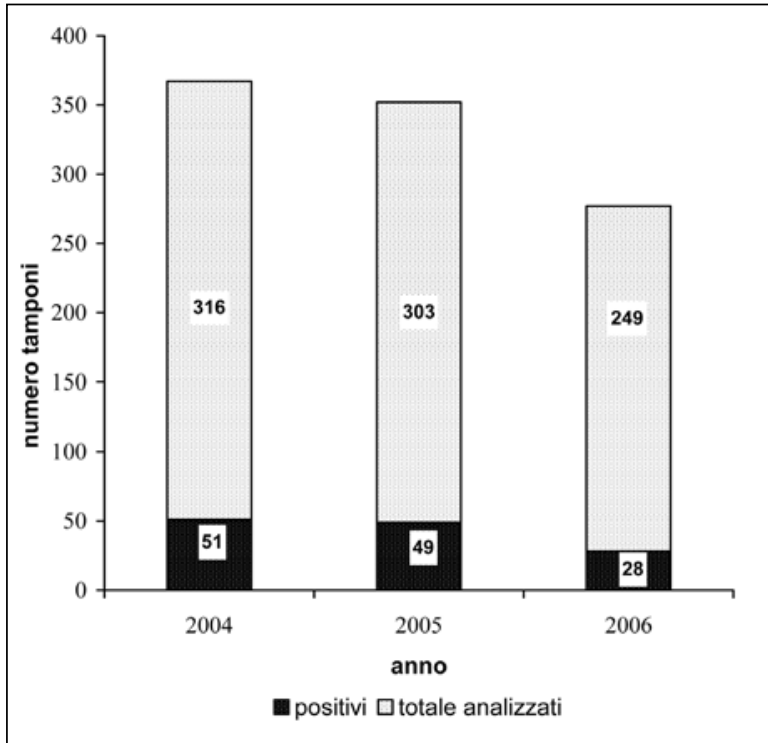

Figura I. Incidenza di S. pyogenes relativamente al totale dei tamponi faringei analizzati in ciascun anno (2004, 2005, 2006).

Tabella I. Percentuali di sensibilità ai macrolidi degli stipiti di S. pyogenes isolati da soggetti adulti e pediatrici nel periodo Novembre 2003-Dicembre 2005.

\begin{tabular}{|c|c|c|c|c|}
\hline \multirow{2}{*}{$\begin{array}{l}\text { Pazienti } \\
\text { (n. ceppi saggiati) }\end{array}$} & \multirow{2}{*}{ Antibiotici } & \multirow{2}{*}{\multicolumn{3}{|c|}{$\begin{array}{lcc}\text { S } & \text { I } & \text { R } \\
\text { Percentuale } & \text { (n. ceppi) }\end{array}$}} \\
\hline & & & & \\
\hline \multirow[t]{3}{*}{ Adulti (45) } & ERY & $88.9 \%(40)$ & $6.7 \%(3)$ & $4.4 \%(2)$ \\
\hline & $C L Y$ & $91.1 \%$ (4I) & $6.7 \%(3)$ & $2.2 \%(I)$ \\
\hline & ROK & $100 \%(45)$ & 0 & 0 \\
\hline \multirow[t]{3}{*}{ Pediatrici (55) } & ERY & $81.8 \%(45)$ & $14.5 \%(8)$ & $3.6 \%(2)$ \\
\hline & $C L Y$ & $96.4 \%$ (53) & $3.6 \%(2)$ & 0 \\
\hline & ROK & $100 \%(55)$ & 0 & 0 \\
\hline \multirow[t]{3}{*}{ TOTALI $(100)$} & ERY & $85 \%$ & $11 \%$ & $4 \%$ \\
\hline & CLY & $94 \%$ & $5 \%$ & $1 \%$ \\
\hline & ROK & $100 \%$ & 0 & 0 \\
\hline
\end{tabular}

Legenda: S: sensibile, I: intermedio R: resistente; ERY: eritromicina, CLY: clindamicina, ROK rokitamicina

Tabella 2. Percentuali di sensibilità ai macrolidi degli stipiti di S. pyogenes isolati da soggetti adulti e pediatrici nel periodo Gennaio 2006 - Settembre 2006.

\begin{tabular}{|c|c|c|c|c|}
\hline \multirow{2}{*}{$\begin{array}{l}\text { Pazienti } \\
\text { (n. ceppi saggiati) }\end{array}$} & \multirow{2}{*}{ Antibiotici } & \multirow{2}{*}{\multicolumn{3}{|c|}{$\begin{array}{lcc}\text { S } & \text { I } & \text { R } \\
\text { Percentuale } & \text { (n. ceppi) }\end{array}$}} \\
\hline & & & & \\
\hline \multirow[t]{3}{*}{ Adulti $(20)$} & ERY & $95 \%(19)$ & 0 & $5 \%(I)$ \\
\hline & CLY & $100 \%(20)$ & 0 & 0 \\
\hline & ROK & $100 \%(20)$ & 0 & 0 \\
\hline \multirow[t]{3}{*}{ Pediatrici (8) } & ERY & $75 \%(6)$ & $25 \%(2)$ & 0 \\
\hline & CLY & $100 \%(8)$ & 0 & 0 \\
\hline & ROK & $100 \%(8)$ & 0 & 0 \\
\hline \multirow[t]{3}{*}{ TOTALI (28) } & ERY & $89.3 \%(25)$ & $7.1 \%(2)$ & $3.6 \%(I)$ \\
\hline & CLY & $100 \%(28)$ & (0) & (0) \\
\hline & ROK & $100 \%$ (28) & (0) & (0) \\
\hline
\end{tabular}

Legenda: S: sensibile, I: intermedio R: resistente; ERY: eritromicina, $C L Y$ : clindamicina, $R O K$ rokitamicina.

\section{CONCLUSIONI}

La percentuale di resistenza ai macrolidi riscontrata nel presente studio nella realtà locale esaminata, si è rivelata relativamente bassa se paragonata ai valori registrati nel nostro paese nel corso del precedente decennio. Tra il 1991 a il 1996, infatti, la percentuale di resistenza all'eritromicina in $S$. pyogenes in Italia è cresciuta fino a raggiungere in alcune aree geografiche valori superiori al $40 \%$, per poi decrescere negli anni successivi $(4-6,12,15-17,34)$.

Lo studio ha inoltre dimostrato che, negli isolati di S. pyogenes con mancata sensibilità ai macrolidi, la resistenza era conferita principalmente da meccanismo di efflusso (fenotipo $M$ ). Dal momento che questo tipo di resistenza non influenza l'attività dei macrolidi a 16 atomi (come la rokitamicina), essi possono in taluni casi fornire un certo vantaggio clinico rispetto alle altre molecole di questa classe.

$\mathrm{E}$ interessante sottolineare che non è stato isolato alcun stipite di $S$. pyogenes con fenotipo cMLS: tuttavia, per confermare questo dato, sarebbe opportuno poter analizzare un maggior numero di ceppi resistenti ai macrolidi.

Una relazione tra l'impiego clinico dei macrolidi e la diffusione della resistenza negli Streptococchi di gruppo A è stato segnalato in diversi studi (2, 67, 13-14, 19-20, 22, 31). L'intervallo di tempo necessario affinché una variazione nell'utilizzo di questi farmaci modifichi in maniera significativa l'antibiotico-sensibilità non è tuttavia prevedibile. Pertanto, studi epidemiologici di sorveglianza come questo sono auspicabili non solo per monitorare l'andamento della resistenza e dei meccanismi coinvolti nella sua insorgenza, ma anche per salvaguardare l'utilizzo clinico dei macrolidi.

\section{BIBLIOGRAFIA}

1. Abu-Gharbieh E, Vasina V, Poluzzi E, De Ponti F. Antibacterial macrolides: a drug class with a complex pharmacological profile. Pharmacol Res 2004; 50: 211-22.

2. Albrich WC, Monnet DL, Harbarth S. Antibiotic selection pressure and resistance in Streptococcus pneumoniae and Streptococcus pyogenes. Emerg Infect Dis 2004; 10: 514-7.

3. Alos JI, Aracil B, Oteo J, Torres C, Gomez-Garces JL. High prevalence of erythromycin-resistant, clindamycin/miocamycin-susceptible (M phenotype) Streptococcus pyogenes: results of a Spanish multicentre study in 1998. Spanish Group for the Study of Infection in the Primary Health Care Setting. J Antimicrob Chemother 2000; 45: 605-9.

4. Avanzini C, Bosio K, Volpe G, Dotti G, Savoia D. Streptococcus pyogenes collected in Torino (northwest Italy) between 1983 and 1998: survey of macrolide resistance and trend of genotype by RAPD. Microb Drug Resist 2000; 6: 289-95.

5. Bassetti M, Manno G, Collida A, et al. Erythromycin 
resistance in Streptococcus pyogenes in Italy. Emerg Infect Dis 2000; 6: 180-3

6. Bassetti M, Mantero E, Gatti G, Di Biagio A, Bassetti D. Streptococcus pyogenes erythromycin resistance in Italy. Emerg Infect Dis 1999; 5: 302-3.

7. Bergman M, Huikko S, Pihlajamaki, et al. Finish Study Group for Antimicrobial Resistance (FiRe Network). Effect of macrolide consumption on erythromycin resistance in Streptococcus pyogenes in Finland in 1997-2001. Clin Infect Dis 2004; 38: 1251-6.

8. Bingen E, Bidet P, Mihaila-Amrouche L, et al. Emergence of macrolide-resistant Streptococcus pyogenes strains in French children. Antimicrob Agents Chemother 2004; 48: 3559-62.

9. Bisno AL, Gerber MA, Gwaltney JM jr., Kaplan EL, Schwartz RH. Diagnosis and managment of group A streptococcal pharyngitis: a pratical guideline. Clin Infect Dis 1997; 25: 574-83.

10. Blasi F. Atypical pathogens and respiratory tract infections. Eur Respir J 2004; 24: 171-81.

11. Brook I, Dohar JE. Management of group A betahemolytic streptococcal pharyngotonsillitis in children. J Fam Pract. 2006; 55: S1-S12.

12. Cellesi C, Chigiotti S, Zanchi A, Mencarelli M, Corbisiero R, Rossolini GM. Susceptibility of macrolide and beta-lactam antibiotics of Streptococcus pyogenes strains isolated over a four-year period in central Italy. J Chemother 1996; 8: 188-92.

13. Cizman M, Pokorn M, Seme K, Orazem A, Paragi M. The relationship between trends in macrolide use and resistance to macrolides of common respiratory pathogens. J Antimicrob Chemother 2001; 47: 475-7.

14. Cizman M, Pokorn M, Seme K, Paragi M, Orazem A. Influence of increased macrolide consumption on macrolide resistance of common respiratory pathogens. Eur J Clin Microbiol Infect Dis 1999; 18: 522-4.

15. Cocuzza CE, Mattina R, Mazzariol A, et al. High incidence of erythromycin-resistant Streptococcus pyogenes in Monza (North Italy) in untreated children with symptoms of acute pharyngo-tonsillitis: an epidemiological and molecular study. Microb Drug Resist 1997; 3: 371-8

16. Cornaglia G, Ligozzi M, Mazzariol A, et al. Resistance of Streptococcus pyogenes to erythromycin and related antibiotics in Italy. The Italian Surveillance Group for Antimicrobial Resistance. Clin Infect Dis. 1998; 27 (Suppl 1): S87-S92.

17. Cornaglia G, Ligozzi M, Mazzariol A, Valentini M, Orefici G, Fontana R. Rapid increase of resistance to erythromycin and clindamycin in Streptococcus pyogenes in Italy, 1993-1995. The Italian Surveillance Group for Antimicrobial Resistance. Emerg Infect Dis 1996; 2: 339-42.

18. Cunningham MV. Pathogenesis of group A Streptococcal infections. Clin Microbiol Rev 2000; 13: 470-511.

19. Gagliotti C, Nobilio L, Milandri M, Moro ML. Emilia-Romagna Antibiotic Resistance Study Group. Macrolide prescriptions and erythromycin resistance of Streptococcus pyogenes. Clin Infect Dis 2006; 42: 1153-6.

20. Garcia-Rey C, Aguilar L, Baquero F, Casal J, Martin JE. Pharmacoepidemiological analysis of provincial differences between consumption of macrolides and rates of erythromycin resistance among Streptococcus pyogenes isolates in Spain. J Clin Microbiol 2002; 40: 2959-63.

21. Giovanetti E, Montanari MP, Mingoia M, Varaldo PE. Phenotypes and genotypes of erythromycin-resistant Streptococcus pyogenes strains in Italy and hetero- geneity of inducibly resistant strains. Antimicrob Agents Chemother 1999; 43: 1935-40.

22. Granizo JJ, Aguilar L, Casal J, Dal-Re R, Baquero F. Streptococcus pyogenes resistance to erythromycin in relation to macrolide consumption in Spain (19861997). J Antimicrob Chemother 2000; 46: 959-64.

23. Kaplan EL. The resurgence of group A Streptococcal infections and their sequele. Eur J Clin Microbiol Infect Dis 1991; 10: 55-7.

24. Kaplan EL, Gooch WM, Notario GF, Craft JC. Macrolide therapy of group A streptococcal pharyngitis: 10 days of macrolide therapy (clarithromycin) is more effective in streptococcal eradication than 5 days (azithromycin). Clin Infect Dis 2001; 32: 1798-802.

25. Kaplan EL, Chhatwal GS, Rohde M. Reduced ability of penicillin to eradicate ingested group A streptococci from epithelial cells: clinical and pathogenetic implications. Clin Infect Dis 2006; 43: 1398-406.

26. Katz L, Ashley GW. Translation and protein synthesis: macrolides. Chem Rev 2005; 105: 499-527.

27. Leclercq R. Mechanisms of resistance to macrolides and lincosamides: nature of the resistance elements and their clinical implications. Clin Infect Dis 2002; 34: 482-92.

28. Retsema J, Fu W. Macrolides: structures and microbial targets. Int J Antimicrob Agents 2001; 18: S3S10.

29. Seppala H, Nissinen A, Jarvinen H, et al. Resistance to erythromycin in group A streptococci. N Engl J Med 1992; 326: 292-7.

30. Seppala H, Nissinen A, Yu Q, Huovinen P. Three different phenotypes of erythromycin-resistant Streptococcus pyogenes in Finland. J Antimicrob Chemother 1993; 32: 885-91.

31. Seppala H, Klaukka T, Vuopio-Varkila J, et al. The effect of changes in the consumption of macrolide antibiotics on erythromycin resistance in group A streptococci in Finland. Finnish Study Group for Antimicrobial Resistance. N Engl J Med 1997; 337: 441-6.

32. Sutcliffe J, Tait-Kamradt A, Wondrack L. Streptococcus pneumoniae and Streptococcus pyogenes resistant to macrolides but susceptible to Clindamycin: a common resistance pattern mediated by an efflux system. Antimicrob Ag Chemother 1996; 40: $1817-24$

33. Sutcliffe J, Grebe T, Tait-Kamradt A, Wondrack L. Detection of erythromycin-resistant determinants by PCR. Antimicrob Agents Chemother 1996; 40: 2562-6.

34. Varaldo PE, Debbia EA, Nicoletti G, et al. Nationwide survey in Italy of treatment of Streptococcus pyogenes pharyngitis in children: influence of macrolide resistance on clinical and microbiological outcomes. Artemis-Italy Study Group. Clin Infect Dis 1999; 29: 869-73. 